\title{
The Impact of Targeting Scientific Reasoning on Student Attitudes about Experimental Physics
}

\author{
N.G. Holmes, Joss Ives and D.A. Bonn \\ Dept. of Physics \& Astronomy, University of British Columbia, 6224 Agricultural Road, \\ Vancouver, BC V6T $1 Z 1$
}

\begin{abstract}
Many course interventions in physics explicitly aim to improve student learning and implicitly aim to improve student attitudes toward physics. In this paper we describe an introductory physics lab course aimed to give students more authentic experiences conducting physics experiments. To evaluate student attitudes toward experimental physics, we analyzed and compared student responses to the Colorado Learning and Attitudes about Science Survey for Experimental Physics (E-CLASS) in the intervention course and in a more traditional one-semester physics lab course. While there were significant negative shifts in attitudes in the traditional course, we found no negative shift in the transformed course. We attribute this difference to deliberately supporting learning goals that focus on understanding the process of science through reflection, iteration, and improvement. We discuss possible reasons for the lack of evidence of significant increases.
\end{abstract}

Keywords: Motivation, epistemology, laboratory instruction, E-CLASS

PACS: 01.40.Fk, 01.40.gb, 01.50.Qb

\section{INTRODUCTION}

It has been shown that students' epistemological stances, their beliefs about how knowledge is created, can influence their learning [1]. Unfortunately, many introductory physics courses observe a decline in students' expert-like attitudes and epistemologies $[2,3]$. Positive attitudinal shifts have been observed, however, in courses with a focus on developing epistemologies (whether explicitly [4] or implicitly [5]) and teaching the process of science [6,7]. Introductory labs, therefore, could be a key opportunity for developing students' attitudes and epistemologies through the experimentation process.

Unfortunately, many students disassociate the activities of expert scientists from their own experiences in a lab [2,8], suggesting that the experimentation involved in traditional labs is not engaging students authentically in the scientific process. In a recent study, these beliefs were mapped onto inauthentic scientific inquiry behaviors such as artificially inflating uncertainties or not correcting systematic errors in an experiment [9]. This is unsurprising when one considers the large set of desired learning outcomes in traditional labs, which are often not made explicit to the students. These span learning physics concepts, developing scientific reasoning abilities, acquiring technical lab skills, understanding measurement and uncertainty, applying real-world connections to in-class material, shifting student attitudes and epistemologies regarding the nature of measurement and science, and much more $[10,11]$. Trying to present this diversity of desired outcomes in a single lab course can lead students to cognitive overload, resulting in exposure to many ideas, but mastery of none. Indeed, many students often find there is simply not enough time to focus on all aspects of the labs [9].

In response to these issues, a first-year physics lab course was restructured to engage students in more authentic experimentation behaviors with a more explicit focus on scientific process, especially the reflective and iterative nature of scientific experimentation and development of models. We aim to evaluate whether the new structure of the course impacted students' perceptions and attitudes about experimental physics, as measured by the Colorado Learning and Attitudes about Science Survey for Experimental Physics (E-CLASS) [12]. The responses will be compared to those of another first-year physics lab course with a more traditional structure.

\section{STRUCTURED QUANTITATIVE INQUIRY LABS}

Compared to a traditional lab course, the Structured Quantitative Inquiry Labs (SQILabs) refocus the scope of the learning outcomes to promote mastery only on data handling, measurement, and uncertainty concepts and experimentation skills [13]. While the experiments are all physics-based, the concepts are not mapped to the physics course content and developing physics concepts is not an explicit goal of the course. Instead, the course aims to develop a number of statistical and 
data handling skills (such as least squares fitting and weighted averages), as well as higher level reasoning and critical thinking skills (such as reflecting on the outcomes of experiments and devising follow-up tests or modifications). The overarching aim of these goals is to develop students' epistemologies about the nature of scientific measurement and uncertainty, and provide opportunities for scaffolded experimental design.

For example, in one lab activity, students iterated measurements of the period of a pendulum to reduce their uncertainty. Students compared the period of the pendulum at two different angles and, as the quality of their measurements improved, began to discern the angle dependence due to the second-order correction to the period. Through experiments like this one, students connect the nature of scientific measurement (through improving precision) to the scientific process (identifying limitations of models based on evidence).

Early in the course, cycles of reflecting, iterating, and improving measurements were explicitly structured and this scaffolding was removed later in the course. Students were assessed based on the quality of their measurement (accuracy and precision) and for demonstrating reflection and iteration throughout their experimentation process in a written lab book. To encourage fruitful collaboration in the lab, students work in small groups, submit work individually, but all group members receive the same grade based on the assessment of one group member's work.

We compare this lab, which we call the Structured Quantitative Inquiry Lab (SQILab), to a more traditional introductory physics lab, one meant to synchronize with and support the physics topics covered in lectures. This lab targeted the development of a large number of skills, but had no explicit goals to develop expert-like scientific reasoning. We thus aim to examine whether deliberately targeting authentic scientific reasoning, supported by associated assessment, has a positive impact on students' attitudes and epistemologies about experimental physics.

\section{METHODS}

Participants were 580 students in two introductory physics courses at the University of British Columbia. Only students who wrote both the pre- and post-survey E-CLASS, did not leave more than 10 items blank throughout both the pre- and post-surveys, and correctly responded to the item, "We use this statement to discard the survey of people who are not reading the questions. Please select 'Agree' for both questions to preserve your answers" were included in the study.

The traditional lab course $(n=453)$ is a singlesemester calculus-based physics course, where the lab is a required component of the course, involving 6 three-hour lab sessions during the term. The SQILab $(n=127)$ is also a single-semester calculus-based physics course, with the lab component consisting of 8 three-hour lab sessions. The SQILab is taken by students seeking an enriched physics curriculum and approximately $25 \%$ of the students intended to major in physics or astronomy, compared to only $2 \%$ in the traditional lab. 97\% of students (across both courses) intended to major in a science discipline. Since students in the SQILab opted to take a more challenging course, it is likely that they entered the course with more expert-like attitudes. Comparisons between the courses, therefore, were made only of paired shifts in attitudes from pre- to post-survey.

Students completed the E-CLASS at the beginning and end of the semester in each course. Students in the SQILab completed the survey during lab time and were offered a small amount of course credit for completion. Students in the traditional lab were asked to complete the survey outside of class time and were offered a small amount of course credit for completion.

\section{The E-CLASS Tool}

The E-CLASS poses questions about 30 concepts in three ways [12]:

1. Students' personal attitudes and beliefs: "What do you think?"

2. Students" views of experts: "What would an experimental physicist say about their research?"

3. Students' views of the importance: "How important for earning a good grade in this class was...?"

All 30 concepts were posed as personal and expert beliefs questions both on pre- and post-surveys, while 23 concepts were also posed for level of importance in the course on post-survey only.

Scores for the personal and expert attitudes were converted from a 5-point Likert scale measure of agreement (where 1 is strongly disagree and 5 is strongly agree) to a binary scale of whether their response was favorable or unfavorable, since concept items were phrased such that favorable expert-like responses varied between agreement or disagreement. The favorable responses for each concept item were validated by surveying a sample of experimental physicists [10].

\section{RESULTS}

Two sets of analyses were carried out on students' responses. First, two univariate ANOVAs were carried out on the personal and expert beliefs items separately, both across time and across courses. An independent samples $t$-test was used to compare students' views of how important the concepts were for earning a good 
grade in each course. The next sections will present these analyses individually.

\section{Personal and Expert Beliefs Items}

Figure 1 shows the average fraction of favorable responses by students at pre- and post-survey on both the personal and expert items. Table 1 shows the significance results of the two ANOVAs. The students in the SQILab held more favorable personal beliefs than the students in the traditional course, in line with the general population differences previously described. There was also a significant shift over time, independent of course, and a significant interaction between course and time. This interaction signifies a significant drop in students' personal beliefs in the traditional lab and a neutral shift in SQILab students' personal beliefs.

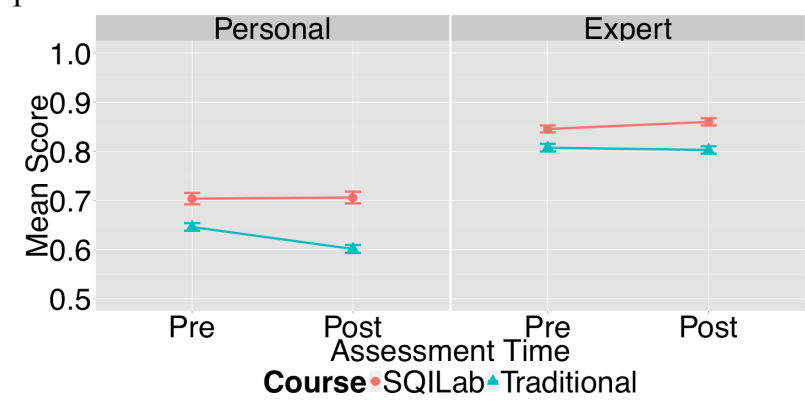

FIGURE 1. Pre-to post-survey shifts for personal and expert beliefs for both courses. Uncertainty bars represent standard uncertainties in the mean.

The population differences were also present in students' expert beliefs. Expert beliefs did not change over time, however, and the interaction between course and time was also non-significant. This stability of students' beliefs about experts was also seen in other courses surveyed with the E-CLASS [10].

TABLE 1. ANOVA table for personal and expert beliefs per course

\begin{tabular}{c|l|c|c|c}
\cline { 1 - 2 } Beliefs & Comparison & \multicolumn{1}{c|}{$d f$} & $\mathrm{~F}$ & $\mathbf{p}^{* * *}$ \\
\cline { 1 - 1 } Personal & Course & 1,578 & 31.20 & $<.001^{* * *}$ \\
& Time & 1,578 & 31.74 & $<.001^{* * *}$ \\
& Course*Time & 1,578 & 10.16 & $.002^{* *}$ \\
\cline { 1 - 3 } Expert & Course & 1,578 & 13.05 & $<.001^{* * *}$ \\
& Time & 1,578 & 0.01 & .931 \\
& Course*Time & 1,578 & 1.74 & .188 \\
\hline
\end{tabular}

\section{Importance for the Course Items}

When comparing the average Likert score for how important the concepts were for obtaining a good grade in the course, the average Likert scores of the SQILab $(M=3.86, S D=0.45)$ students were significantly higher than those of the traditional lab students $(M=$
$3.67, S D=0.58), t(254)=4.12, p<.001$. This suggests that the assessment in the SQILab more successfully addressed the items on the E-CLASS.

\section{DISCUSSION}

This study examined the effects of two lab courses on student attitudes and beliefs about experimental physics. It was found that students in a traditional lab demonstrated significant negative shifts on personal attitudes, consistent with other measures of student attitudes deteriorating over an introductory physics course [2,3]. While positive shifts in epistemologies have been found in courses that emphasized scientific process [4-7], the results presented here suggest that simply engaging in experimentation, as in the traditional course, does not produce the same effect. From this result, one could conclude that traditional lab experiments do not explicitly engage students in the scientific process. Without explicitly supporting the scientific reasoning goals, students' attention is not exclusively focused there.

The goals and structure of the SQILab went beyond measurement and uncertainty by also emphasizing reflection, iteration, and improvement of experimental methods and physical models. These are vital for developing expert-like epistemologies and attitudes toward experimental physics. Through these cycles, students saw physics models adapt and change as the quality of their measurements improved. The item, "When doing a physics experiment, I don't think much about sources of systematic error" was addressed in experiments such as the pendulum measurement described earlier, where students had to confront whether their measurements disagreed with a given theory due to systematic errors in their measurements or systematic deviations in the theory. More students in the SQILab responded favorably on this item by the end of the term (from $73 \%$ at pre-survey to $82 \%$ at post-survey), while fewer students did in the traditional lab (from $67 \%$ to $55 \%$ ).

These cycles also moved uncertainties from abstract calculations to important tools that were useful for understanding their data. Indeed, the item, "Calculating uncertainties usually helps me understand my results better" is a key goal targeted by the reflection and iteration cycles described earlier, but one that might be regarded as an implicit goal in traditional courses as well. This item showed an increase in favorable responses in the SQILab (from $61 \%$ of students responding favorably to $77 \%$ ) and a decrease for the traditional lab (from $64 \%$ to $47 \%$ ).

Finally, the course had a large focus on data handling and analysis procedures. The item "If I don't have clear directions for analyzing data, I am not sure how to choose an appropriate analysis method" showed 
an increase in favorable responses in the SQILab (from $34 \%$ to $44 \%)$ and no shift in the traditional lab $(25 \%$ favorable responses at pre- and post-survey).

Course elements such as these provided students in the SQILab with more authentic scientific reasoning experiences. Even with this focus, however, students in the SQILab did not improve significantly on their expert-like beliefs overall. This may be due to a subset of items on the E-CLASS that were not targeted in the course. For example, the course deliberately does not involve much experimental design and students do not do experiments that come from their own research questions. While the reflect, iterate, and improve cycles provide opportunities for designing the improvements to their measurements, the item, "When doing an experiment I usually think up my own questions to investigate" was deliberately not targeted. Indeed, this item shows a decrease in favorable responses in the SQILab (from $37 \%$ to $31 \%$ ) and in the traditional course (from $32 \%$ to $20 \%$ ).

Another possible reason for the lack of overall significant upward shifts in the SQILab may be that our goals to develop students' scientific reasoning were insufficient to address their attitudes and epistemologies. Significant shifts may require explicit targeting, rather than implicitly addressing them in activities [4]. While our course more thoroughly engaged students in the scientific process, the course did not explicitly address their beliefs and attitudes. This explicit attention could be in the form of activities to reflect on the nature of science, on students' own learning, on the learning of their peers, and on the learning of scientists [7]. The latter, in particular, would help address epistemological issues and connect students' experiences in physics class with the nature of physics more broadly.

It is important to be careful when comparing this study to those mentioned above. In particular, the context of epistemologies in the lab, versus those in lecture courses, is certainly a distinct experience for students. The epistemological concepts addressed by the E-CLASS differ significantly from the surveys used in the other studies. It is also a newer instrument so there is not yet a large database of results of other courses and other populations.

\section{CONCLUSION}

This study has clarified the distinction between engaging students in authentic scientific process in a lab and engaging students in experimentation. Simply performing physics experiments does not improve students' attitudes and epistemologies about experimental physics. While the SQILab demonstrates an approach that yields improvement over the traditional course, deliberate and explicit attention to students' attitudes and epistemologies may be required.

The study also demonstrates the importance of aligning assessment to the desired learning outcomes. Students in the SQILab viewed the items on the ECLASS as more important for earning a good grade in the course than the students in the traditional course. With the goals aligned with assessment, students knew where to focus their attention, thus maintaining their attitudes in those areas.

\section{ACKNOWLEDGMENTS}

We would like to acknowledge the support and advice from Heather Lewandowski, Takako Hirokawa, Carl Wieman, Sarah Gilbert, and James Day. This research was supported by UBC's Carl Wieman Science Education Initiative.

\section{REFERENCES}

1. L. Lising, and A. Elby, Am. J. Phys. 73(4), 372-382 (2005).

2. E.F. Redish, J.M. Saul, and R.N. Steinberg, Am. J. Phys. 66(3), 212-224 (1998)

3. W.K. Adams, K.K. Perkins, N.S. Podolefsky, M. Dubson, N.D. Finkelstein, and C.E. Wieman, Phys. Rev. ST Phys. Educ. Res. 2(1), 010101 (2006)

4. A. Elby, Am. J. Phys. 69(S1), S54-S64 (2001)

5. B.A.Lindsey, L. Hsu, H. Sadaghiani, J.W. Taylor, and K. Cummings, Phys. Rev. ST Phys. Educ. Res. 8, 010102 (2012)

6. E. Brewe, L. Kramer, and G. O'Brien. Phys. Rev. ST Phys. Educ. Res, 5(1), 013102 (2009)

7. V.K. Otero, and K.E. Gray, Phys. Rev. ST Phys. Educ. Res, 4(2), 020104 (2008)

8. A. Buffler, F. Lubben, and B. Ibrahim, Int. J. Sci. Educ. 31(9), 1137-1156 (2009)

9. N.G. Holmes and D.A. Bonn, in 2013 PERC Proceedings, Portland, OR, 2013, edited by P. V. Engelhardt, A. D. Churukian, and D. L. Jones, AIP Conf. Proc, 185-188

10. B.M. Zwickl, N. Finkelstein, and H. J. Lewandowski. Am. J. Phys. 81(1), 63-70 (2013)

11. American Association of Physics Teachers, Am. J. Phys. 66(6) (1998).

12. B.M. Zwickl, N.D. Finkelstein, and H.J. Lewandowski, in 2012 PERC Proceedings, Philadelphia, PA, 2012, edited by P. V. Engelhardt, A. D. Churukian, and N. S. Rebello, AIP Conf. Proc. 1513, 442-445

13. J. Day, and D.A. Bonn, Phys. Phys. Rev. ST Phys. Educ. Res, 7(1), 010114 (2011) 